\title{
CHAOS IN RELATIVITY AND COSMOLOGY
}

\author{
G. CONTOPOULOS ${ }^{1,2}$, N. VOGLIS ${ }^{2}$ and C. EFTHYMIOPOULOS ${ }^{1,2}$ \\ ${ }^{1}$ Research Center for Astronomy, Academy of Athens \\ ${ }^{2}$ Department of Astronomy, University of Athens
}

\begin{abstract}
Chaos appears in various problems of Relativity and Cosmology. Here we discuss (a) the Mixmaster Universe model, and (b) the motions around two fixed black holes. (a) The Mixmaster equations have a general solution (i.e. a solution depending on 6 arbitrary constants) of Painlevé type, but there is a second general solution which is not Painlevé. Thus the system does not pass the Painlevé test, and cannot be integrable. The Mixmaster model is not ergodic and does not have any periodic orbits. This is due to the fact that the sum of the three variables of the system $(\alpha+\beta+\gamma)$ has only one maximum for $\tau=\tau_{m}$ and decreases continuously for larger and for smaller $\tau$. The various Kasner periods increase exponentially for large $\tau$. Thus the Lyapunov Characteristic Number (LCN) is zero. The "finite time LCN" is positive for finite $\tau$ and tends to zero when $\tau \rightarrow \infty$. Chaos is introduced mainly near the maximum of $(\alpha+\beta+\gamma)$. No appreciable chaos is introduced at the successive Kasner periods, or eras. We conclude that in the Belinskii-Khalatnikov time, $\tau$, the Mixmaster model has the basic characteristics of a chaotic scattering problem. (b) In the case of two fixed black holes $M_{1}$ and $M_{2}$ the orbits of photons are separated into three types: orbits falling into $M_{1}$ (type I), or $M_{2}$ (type II), or escaping to infinity (type III). Chaos appears because between any two orbits of different types there are orbits of the third type. This is a typical chaotic scattering problem. The various types of orbits are separated by orbits asymptotic to 3 simple unstable orbits. In the case of particles of nonzero rest mass we have intervals where some periodic orbits are stable. Near such orbits we have order. The transition from order to chaos is made through an infinite sequence of period doubling bifurcations. The bifurcation ratio is the same as in classical conservative systems.
\end{abstract}

\section{Introduction}

(a) The subject of chaos in Relativity and Cosmology has attracted much interest in recent years. It started with the papers on the Mixmaster Universe model by Belinskii and Khalatnikov (1969) and independently by Misner (1969). But, despite the large number of papers written on this subject (Hobill et al 1994 and references therein), this problem has not been completely solved up to now.

Some recent results will be discussed in the next sections. Perhaps the most impressive result is that this model is not ergodic, nevertheless it is chaotic in the sense of chaotic scattering.

(b) A problem in General Relativity, where chaos is dominant, is the case of two fixed black holes (Contopoulos 1990, 1991). This problem will be discussed in Section 5 below. It is remarkable that the relativistic problem is chaotic, while the corresponding classical problem of two fixed centers is completely integrable.

Among other problems in Relativity and Cosmology, where chaos plays a role, are the following.

(c) Chaos in Special Relativity (Drake et al. 1996). Chaos was found in the motion of charged particles in a static electric field.

(d) A spinning particle in the Schwarzschild spacetime (Suzuki and Maeda 1997). The Schwarzschild spacetime is a 1-dimensional system, hence it is integrable. But adding further degrees of freedom can make the problem chaotic. 
Chaos in perturbed Schwarzschild spacetimes has been found by Bombelli \& Calzetta (1992) and by Letelier \& Vieira (1997).

(e) The Robertson-Walker spacetime is also a 1-dimensional system (spherical), hence integrable. But the addition of another field makes it non- integrable and chaotic (Calzetta et al. 1993, Cornish \& Shellard 1998).

(f) Motion in the field generated by gravitational waves (Varvoglis \& Papadopoulos 1992, Chicone et al. 1997, Podolsky \& Vesely 1998).

(g) Chaos in Yang-Mills fields of curved spacetime (Darian \& Künzle 1996, Barrow \& Levin 1998).

In the present paper we will discuss only the first two topics, namely the Mixmaster model and the case of two fixed black holes.

\section{The Mixmaster Universe Model.}

This model is a particular solution of Einstein's field equations (e.g. Landau \& Lifshitz, 1971) that leads to three second order differential equations for three scale factors $\alpha, \beta$ and $\gamma$ :

$$
2 \ddot{\alpha}=\left(e^{2 \beta}-e^{2 \gamma}\right)^{2}-e^{4 \alpha}
$$

and cyclic permutations of it, combined with a zero-energy constrain:

$$
\begin{aligned}
H \equiv & \frac{1}{16}\left(p_{\alpha}^{2}+p_{\beta}^{2}+p_{\gamma}^{2}-2 p_{\alpha} p_{\beta}-2 p_{\beta} p_{\gamma}-2 p_{\alpha} p_{\gamma}\right) \\
& +e^{4 \alpha}+e^{4 \beta}+e^{4 \gamma}-2 e^{2(\alpha+\beta)}-2 e^{2(\beta+\gamma)}-2 e^{2(\alpha+\gamma)}=0,
\end{aligned}
$$

where

$$
p_{\alpha}=-4(\dot{\beta}+\dot{\gamma}), \quad p_{\beta}=-4(\dot{\gamma}+\dot{\alpha}), \quad p_{\gamma}=-4(\dot{\alpha}+\dot{\beta}) .
$$

The dots are derivatives with respect to the Belinskii- Khalatnikov time $\tau=-\ln t$, where $t$ is cosmological time. Thus when the cosmological time $t$ goes to zero at the big bang, the time $\tau$ goes to infinity.

The Mixmaster equations can be derived from the Hamiltonian (2):

$$
\dot{\alpha}=\left(p_{\alpha}-p_{\beta}-p_{\gamma}\right) / 8, \quad \dot{p}_{\alpha}=-4\left[e^{4 \alpha}-e^{2(\alpha+\beta)}-e^{2(\alpha+\gamma)}\right],
$$

and cyclic permutations of them.

Another simple set of variables is

$$
X=e^{2 \alpha}, \quad Y=e^{2 \beta}, \quad Z=e^{2 \gamma}, \quad p_{X}=p_{\alpha} / 4, \quad p_{Y}=p_{\beta} / 4, \quad p_{Z}=p_{\gamma} / 4
$$

In these variables the equations of motion take the simple form

$$
\dot{X}=X\left(p_{X}-p_{Y}-p_{Z}\right), \quad \dot{p}_{X}=X(Y+Z-X),
$$


and cyclic permutations of them, while the zero energy constrain is

$$
\begin{aligned}
E \equiv & p_{X}^{2}+p_{Y}^{2}+p_{Z}^{2}-2 p_{X} p_{Y}-2 p_{Y} p_{Z}-2 p_{X} p_{Z} \\
& +X^{2}+Y^{2}+Z^{2}-2 X Y-2 Y Z-2 Z X=0
\end{aligned}
$$

Numerical integrations of the Mixmaster equations strongly suggest that the Lyapunov characteristic number is zero (Hobill et al. 1992).

This, and other theoretical indications, lead to the view that the Mixmaster model is not chaotic (Cushman and Sniatycki 1995). However other indications lead to the conclusion that the Mixmaster is chaotic.

In the present paper we will see how these two views can be made compatible.

One method to find evidence for integrability of a dynamical system is the Painlevé test (Ablowitz et al. 1980). If all solutions of the equations of motion have only poles as movable singularities, then probably the system is integrable.

The first general solution of Eqs. (7), i.e. a solution that depends on 6 arbitrary constants, which satisfies the Painlevé condition, was given by Contopoulos, Grammaticos and Ramani (1993). It is given as Laurent series

$$
\begin{aligned}
& X= \pm \frac{i}{s}+\delta_{1} s+\ldots, \quad Y=x_{2} s+\ldots, \quad Z=x_{3} s+\ldots, \\
& p_{X}=-\frac{1}{s}+\ldots, \quad p_{Y}=p_{2}+\ldots, \quad p_{Z}=p_{3}+\ldots,
\end{aligned}
$$

where $s=\tau-\tau_{0}$, and the arbitrary constants are $\delta_{1}, x_{2}, x_{3}, p_{2}, p_{3}, \tau_{0}$.

However it was found later that this is not the only general solution of the Mixmaster model. Another solution found by Latifi et al. (1994), by perturbing the well known Taub solution (Taub 1951), does not have the Painlevé property. The same solution was derived by Contopoulos, Grammaticos and Ramani (1995) by starting with a different solution of the Mixmaster equations (6):

$$
\begin{aligned}
X & =A+C A^{2} s+\ldots, & Y=Z=\frac{1}{A s^{2}}+\ldots, \\
p_{X} & =\frac{2}{s}+A C+A D s+\ldots, & p_{Y}=p_{Z}=\frac{1}{s}+. .
\end{aligned}
$$

that depends on only 4 arbitrary constants $\left(A, C, D, \tau_{0}\right)$. This solution passes the Painlevé test to all orders, but it is special because $Y=Z$ and $p_{Y}=p_{Z}$. In order to find a more general solution we set

$$
\delta=Y-Z, \quad q=p_{Y}-p_{Z}
$$

and find expressions for $\delta$ and $q$ that contain exponential singularities (Contopoulos et al. 1995). This general solution (depending on 6 arbitrary constants) is not of Painlevé type. Therefore the Mixmaster model does not pass the Painlevé test, although it has one general solution that is of Painlevé type. As a conclusion the Mixmaster model cannot be integrable with two more integrals besides the energy. 
On the other hand Cushman and Sniatycki (1995) noted that the Mixmaster model is not mixing, nor even ergodic. This conclusion is based on a simple equation for the quantity

$$
\Omega=e^{-2(\alpha+\beta+\gamma)},
$$

that can be easily derived from Eqs.(7), namely

$$
\ddot{\Omega}=\Omega\left[\left(p_{\alpha}^{2}+p_{\beta}^{2}+p_{\gamma}^{2}\right) / 8-E\right] .
$$

An equivalent equation was first derived from the Raychaudhuri (1955) equation (Rugh and Jones 1990; see also Contopoulos et al. 1995).

In Eq. (12) for $E \leq 0$ (and in particular for the Mixmaster model where $E=0$ ), the second derivative $\ddot{\Omega}$ has always the same sign as $\Omega$. As a consequence $\Omega$ has only one minimum and tends to infinity as $\tau$ goes to $\pm \infty$. This means that $(\alpha+\beta+\gamma)$ has one maximum at a certain time $\tau=\tau_{m}$ and decreases monotonically, both for larger $\tau$ and for smaller $\tau$ (Fig.1). The quantity $(\dot{\alpha}+\dot{\beta}+\dot{\gamma})$ is negative for $\tau>\tau_{m}$ and positive for $\tau<\tau_{m}$. Thus the values of $(\alpha, \beta, \gamma, \dot{\alpha}, \dot{\beta}, \dot{\gamma})$ cannot come back to their initial values, or close to them. Two conclusions are derived from this fact.

(1) The Mixmaster model does not have any recurrence, therefore it is not mixing, nor ergodic, despite its name (Cushman and Sniatycki 1995).

(2) The Mixmaster model (with $E=0$ ) does not have any periodic orbits.

However, the usual notions of ergodicity etc. apply to compact systems, while the Mixmaster model is not compact. As we have noticed already (Contopoulos et al. 1995), the Mixmaster model behaves like a chaotic scattering system.

Christiansen et al.(1995) have found unstable periodic orbits in the case $E>0$, but this is not the Mixmaster case, which has $E=0$. Cornish and Levin $(1997,1998)$ have found many unstable periodic orbits in a projected subset of the Mixmaster phase space. The study of these periodic orbits strongly suggests a fractal structure on this subset, and this is a signature of chaos.

Our approach is different. We deal with the full phase space of the Mixmaster model and try to find the sources of chaos in it.

\section{A Numerical Example.}

The fact that $\Omega$ goes to infinity both for $\tau \rightarrow \infty$ and for $\tau \rightarrow-\infty$ implies that the quantity $(\alpha+\beta+\gamma)$ goes to minus infinity for $\tau \rightarrow \pm \infty$ (Fig.1).

This implies that at least one of the quantities $(\alpha, \beta, \gamma)$ goes to minus infinity. But this happens in an oscillatory way, i.e. the quantities $(\alpha, \beta, \gamma)$ are unbounded, but there is no finite time $\tau$, beyond which any of these quantities remains smaller than a given negative number.

As a numerical example we take an orbit with initial conditions: $\alpha, \beta, \gamma, p_{\alpha}, p_{\beta}, p_{\gamma}$ $=\ln 2 / 2,0,0,0,0,8$. In this model the function $(\alpha+\beta+\gamma)$ (Fig.1) has a maximum 0.60 for $\tau=\tau_{m}=-0.48$, i.e. very close to $\tau=0$. 


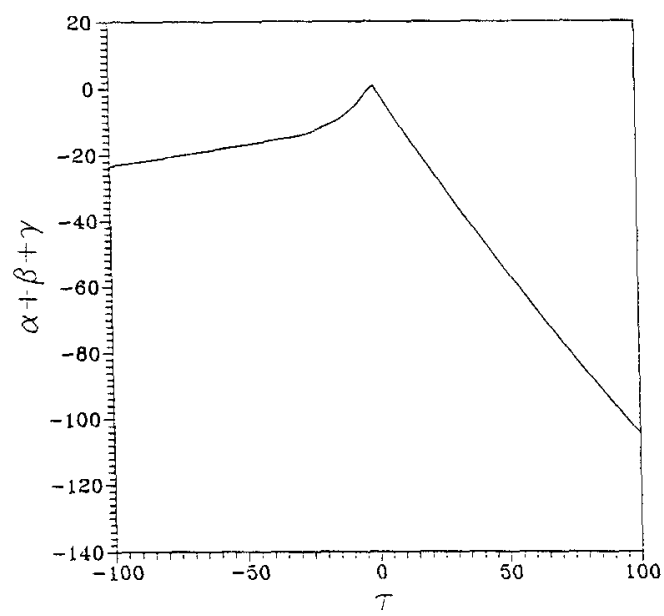

Fig. 1. The variation of $(\alpha+\beta+\gamma)$ as a function of time, for an orbit with initial conditions $\alpha=\ln 2 / 2, \beta=\gamma=p_{\alpha}=p_{\beta}=0, p_{\gamma}=8$.
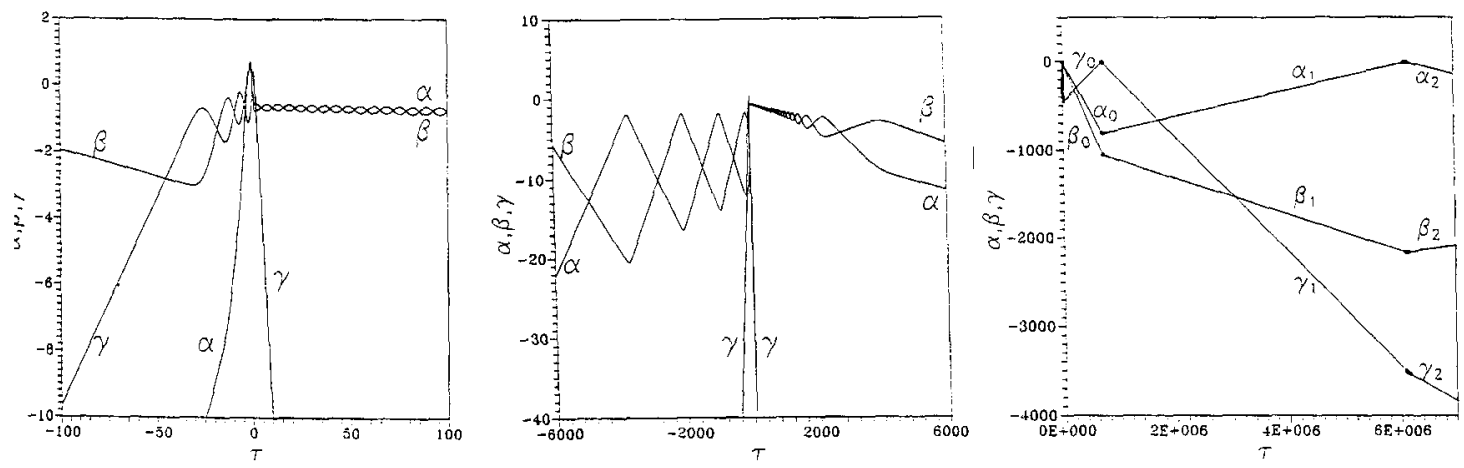

Fig. 2. The variations of $\alpha, \beta, \gamma$ in time $\tau$, (a) from $\tau=-100$ to $\tau=100$ (b) From $\tau=-6000$ to $\tau=6000$ (c) From $\tau=0$ to $\tau=7 \times 10^{8}$. Initial conditions as in Fig.1.

In Fig. 2a,b,c we see the variation of the quantities $(\alpha, \beta, \gamma)$ in time, both in the positive and in the negative time direction.

As $\tau$ increases from $\tau_{m}$ the value of $\gamma$ decreases abruptly. At the same time $\alpha$ and $\beta$ undergo oscillations, first with small period (Fig. 2a) and later with larger and larger period (Figs. $2 \mathrm{~b}, \mathrm{c})$. The value of $\gamma$ becomes minimum $(\gamma \approx-460)$ for $\tau \approx 3500$, and then increases to a maximum close to zero at $\tau \approx 8 \times 10^{5}$. Later on it decreases and increases an infinite number of times.

If $\tau$ decreases from $\tau_{m}, \gamma$ undergoes some oscillations together with $\beta$ (Fig. 2a), while $\alpha$ goes first to a maximum and then decreases. For more negative $\tau$ the value of $\gamma$ decreases considerably, while $\alpha$ and $\beta$ undergo oscillations (Fig. 2b). 
If we exclude a certain interval around $\tau=0$ (say from $\tau=-100$ to $\tau=$ +6000 ) the quantities $\alpha, \beta, \gamma$ are in general very small, so that the exponential members of Eqs. (5) are negligible. Then $p_{\alpha}, p_{\beta}, p_{\gamma}$ and $\dot{\alpha}, \dot{\beta}, \dot{\gamma}$ are almost constant, i.e. the variations of $\alpha, \beta, \gamma$ are almost linear, except when one of them becomes maximum near zero. In that case a rather abrupt change of the inclination of all three lines $\alpha, \beta, \gamma$, occurs (Fig. 2c).

Every period, in which $\dot{\alpha}, \dot{\beta}, \dot{\gamma}$ are almost constant, is called a Kasner period (Misner et al. 1977, Khalatnikov et al. 1985). We will prove (section 4) that for $\tau$ beyond $\tau_{m}$ two derivatives among $\dot{\alpha}, \dot{\beta}, \dot{\gamma}$ are negative and one positive. At every transition from one Kasner period to the next two derivatives (e.g. $\dot{\alpha}$ and $\dot{\beta}$ ) change sign, while the third derivative remains negative.

Similar transitions occur for $\tau<\tau_{m}$. In this case two of the quantities $\dot{\alpha}, \dot{\beta}, \dot{\gamma}$ are positive and one negative.

In order to find the chaotic properties of the orbits we solve the variational equations of Eqs. (5). Namely if we consider a deviation $\xi$ with coordinates $\left(\Delta \alpha, \Delta \beta, \Delta \gamma, \Delta p_{\alpha}, \Delta p_{\beta}, \Delta p_{\gamma}\right)$ we have the equations

$$
\begin{aligned}
\dot{\Delta \alpha} & =\frac{1}{8}\left(\Delta p_{\alpha}-\Delta p_{\beta}-\Delta p_{\gamma}\right), \\
\Delta \dot{p}_{a} & =-8\left[\left(2 e^{4 \alpha}-e^{2(\alpha+\beta)}-e^{2(\alpha+\gamma)}\right) \Delta \alpha-e^{2(\alpha+\beta)} \Delta \beta-e^{2(\alpha+\gamma)} \Delta \gamma\right],
\end{aligned}
$$

and cyclic permutations of them.

The variational equations (13) are solved, together with the equations of motion, and give the variation

$$
|\xi|=\left[\Delta \alpha^{2}+\Delta \beta^{2}+\Delta \gamma^{2}+C^{2}\left(\Delta p_{\alpha}^{2}+\Delta p_{\beta}^{2}+\Delta p_{\gamma}^{2}\right)\right]^{1 / 2}
$$

where $C$ is a constant, having the dimension of time. In the present paper we take $C=1$. Then we find the "finite time Lyapunov characteristic number"

$$
L_{t}=\frac{\ln \left|\xi / \xi_{0}\right|}{\tau}
$$

whose limit for $\tau \rightarrow \infty$ is the Lyapunov characteristic number LCN. We calculate also the "local LCN" (or stretching number)

$$
a_{i}=\frac{\ln \left|\xi_{i+1} / \xi_{i}\right|}{\Delta \tau}
$$

at every step $\Delta \tau$ (Voglis and Contopoulos 1994, Smith and Contopoulos 1996).

We start with two different deviations $\xi_{0}$ at the initial time $\tau=-15000$. In Fig.3a,b we give the stretching number $a$ for the cases $\xi_{0}=(1,0,0,0,0,0)$ and $\xi_{0}=(0,1,0,0,0,0)$ as functions of $\tau$.

Between $\tau=-15000$ and $\tau=-100 a$ is very close to zero. Between $\tau=-100$ and $\tau=+100 a$ undergoes large variations, especially neat $\tau=0$, but it is positive most of the time (Fig.3a). Beyond $\tau=100$ the values of $a$ are again close to zero, 

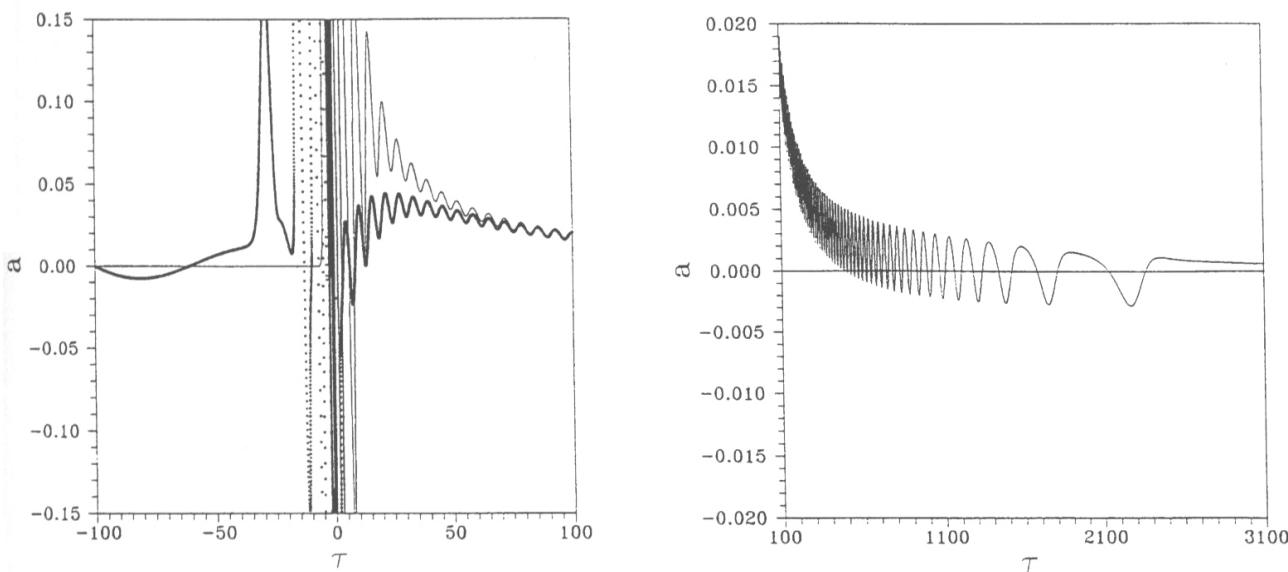

Fig. 3. (a)The variation of the stretching number $a$ as a function of $\tau$ for the orbit of Fig. 1 and initial variations at $\tau=-15000: \xi=(1,0,0,0,0,0)$ (continuous line) and $\xi=(0,1,0,0,0,0)$ (dots, or heavy line) from $\tau=-100$ to $\tau=+100$. (b) The same functions $a(\tau)$ from $\tau=100$ to $\tau=3100$ almost coincide.

but with small scale variations(Fig.3b). The two initial conditions give different curves $a(\tau)$, but for large $\tau$ the two curves come very close to each other.

In Fig.4a,b,c,d we give the spectrum of $a$ for different time intervals. When $\tau$ is between $\tau=-15000$ and $\tau=-1000$ (Fig.4a) the spectrum of $a$ is peaked around $a=0$, like a delta function. Thus no chaos appears during that period. Between $\tau=-1000$ and $\tau=-100$ (Fig.4b) the spectrum has a small positive part for both initial conditions. Between $\tau=-100$ and $\tau=+100$ (Fig.4c) the spectrum has relatively large positive parts. The two spectra are different, but the average value of $a$ is almost the same: $\langle a\rangle=4 \times 10^{-2}$. Therefore the "finite time LCN" during that period is positive. For larger positive times (between $\tau=100$ and $\tau=15000$, Fig.4d) the spectrum approaches again a delta function around $a=0$. The average value of $a$ for large $\tau$ tends to zero. (E.g. in the time interval of Fig. $4 \mathrm{~d}$ it is $\langle a\rangle=4 \times 10^{-4}$ ). Thus the usual LCN is probably zero.

We conclude that the finite time Lyapunov characteristic number is significantly positive only during a period around $\tau=\tau_{m}$, when $(\alpha+\beta+\gamma)$ is maximum. For large positive and negative times the finite time $\mathrm{LCN}$ goes to zero.

Thus chaos appears in the Mixmaster model when $(\alpha+\beta+\gamma)$ is close to its maximum, that is when $\alpha, \beta, \gamma$ are close to zero.

\section{Theoretical Explanation}

When $\alpha, \beta, \gamma$ are negative with absolutely large values, the second members of Eqs. (1) are very close to zero. Thus $\ddot{\alpha}=\ddot{\beta}=\ddot{\gamma}=0$, i.e. $\dot{\alpha}, \dot{\beta}, \dot{\gamma}$ are almost 

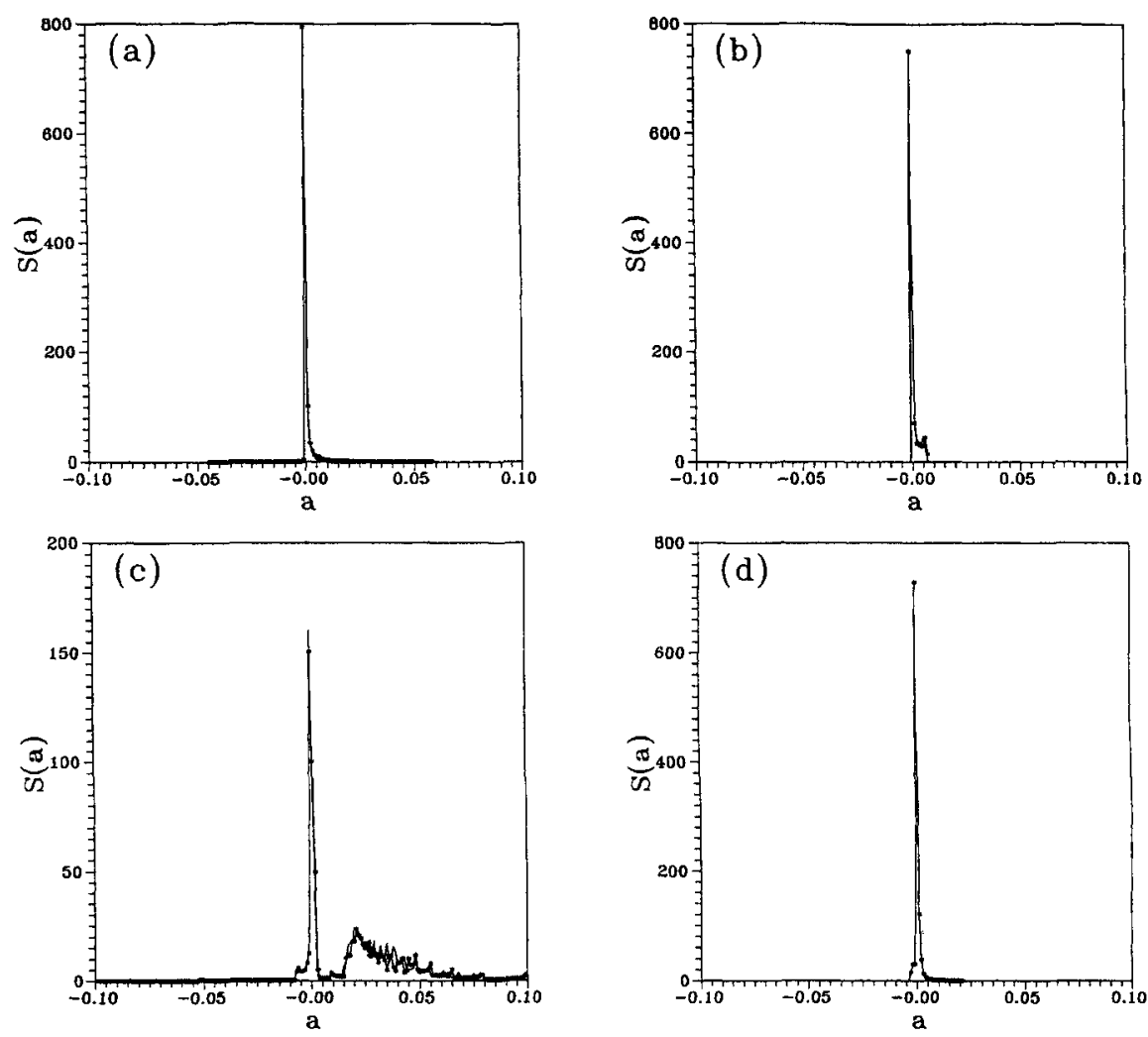

Fig. 4. Spectra of the values of $a$ for the initial conditions of Fig.3, (a)from $\tau=-15000$ to $\tau=-1000$, (b) from $\tau=-1000$ to $\tau=-100$, (c) from $\tau=-100$ to $\tau=+100$, (d) from $\tau=+100$ to $\tau=15000$.

constant and $\alpha, \beta, \gamma$ vary linearly in time. The zero energy constraint is written

$$
-4(\dot{\alpha} \dot{\beta}+\dot{\beta} \dot{\gamma}+\dot{\gamma} \dot{\alpha})=0 \text {. }
$$

For $\tau>\tau_{m}$ we have also

$$
\dot{\alpha}+\dot{\beta}+\dot{\gamma}<0 \text {. }
$$

Equation (17) cannot be satisfied if all three $\alpha, \beta, \gamma$ are positive, or negative. Thus at least one of these quantities is positive, and one negative. Let $\dot{\gamma}>0$ and $\dot{\alpha}<0$. Then we will prove that $\dot{\beta}$ is also negative.

In fact from Eq. (17) we derive

$$
\dot{\gamma}=-\dot{\alpha} \dot{\beta} /(\dot{\alpha}+\dot{\beta}) \text {. }
$$

Thus, unless $\dot{\beta}<0$, we must have $\dot{\beta}>0$ and $\dot{\alpha}+\dot{\beta}>0$. But then Eq.(18) cannot be satisfied. Therefore $\dot{\beta}<0$ is the only possible solution. As a conclusion for $\tau>\tau_{m}$ two quantities among $\dot{\alpha}, \dot{\beta}, \dot{\gamma}$, are negative and one positive. 
In a similar way we prove that for $\tau<\tau_{m}$ two quantities are positive and one negative.

Let us consider now a case with $\dot{\gamma}=\dot{\gamma}_{0}>0, \dot{\alpha}=\dot{\alpha}_{0}<0, \dot{\beta}=\dot{\beta}_{0}<0$ and $\dot{\alpha}_{0}+\dot{\gamma}_{0}<0$. (Fig. 2c). As $\gamma$ increases, it reaches values close to zero, while $\alpha, \beta$ are negative with large absolute values. Thus from Eqs.(1) we derive (Landau and Lifshitz 1971,§113):

$$
2 \ddot{\alpha}=2 \ddot{\beta}=e^{4 \gamma}, \quad 2 \ddot{\gamma}+e^{4 \gamma}=0 .
$$

The last of these equations gives

$$
\dot{\gamma}^{2}+e^{4 \gamma} / 4=\dot{\gamma}_{0}^{2}+e^{4 \gamma_{0}} / 4=R^{2} / 4,
$$

where $R$ is a constant, $\gamma_{0}<0$, and $e^{4 \gamma_{0}}$ is very small, hence $\dot{\gamma}_{0} \approx R / 2$.

The maximum $\gamma$ is slightly above zero

$$
\gamma=\ln R / 2
$$

and then $\dot{\gamma}=0$. Later $\gamma$ is again negative and the limiting value of $\dot{\gamma}$ is

$$
\dot{\gamma}_{1}=-R / 2=-\dot{\gamma}_{0} \text {. }
$$

Thus the transition through the maximum value of $\gamma$ is approximately a reflection. At the same time $\dot{p}_{\alpha}$ and $\dot{p}_{\beta}$ are almost zero, i.e. $p_{\alpha}$ and $p_{\beta}$ remain almost constant. Thus we have approximately

$$
\dot{\beta}_{1}+\dot{\gamma}_{1}=\dot{\beta}_{0}+\dot{\gamma}_{0}, \quad \dot{\alpha}_{1}+\dot{\gamma}_{1}=\dot{\alpha}_{0}+\dot{\gamma}_{0},
$$

where $\dot{\beta}_{0}<\dot{\alpha}_{0}$ (Fig.2c). Hence

$$
\dot{\beta}_{1}=\dot{\beta}_{0}+2 \dot{\gamma}_{0}, \quad \dot{\alpha}_{1}=\dot{\alpha}_{0}+2 \dot{\gamma}_{0},
$$

and $\dot{\beta}_{1}<\dot{\alpha}_{1}$. As $\dot{\gamma}_{1}<0$ we must have $\dot{\beta}_{1}<0<\dot{\alpha}_{1}$.

The interval during which $\dot{\gamma}=\dot{\gamma}_{0}>0$, is a Kasner period. In the next Kasner period $\dot{\alpha}_{1}>0$ while $\dot{\beta}_{1}$ and $\dot{\gamma}_{1}$ are negative, and we observe (Fig. 2c) that $\dot{\gamma}_{1}<$ $\dot{\beta}_{1}<0$. Then comes a period where (Fig.2c)

$$
\dot{\alpha}_{2}=-\dot{\alpha}_{1}, \quad \dot{\beta}_{2}=\dot{\beta}_{1}+2 \dot{\alpha}_{1}, \quad \dot{\gamma}_{2}=\dot{\gamma}_{1}+2 \dot{\alpha}_{1},
$$

As long as $\dot{\gamma}$ is smaller than $\dot{\alpha}$ and $\dot{\beta}$ we have oscillations between $\alpha$ and $\beta$. But the value of $\dot{\gamma}$ increases at every Kasner period and after several Kasner periods it becomes positive. Then $\gamma$ becomes maximum and we say that a Kasner era is completed. From then on $\gamma$ undergoes oscillations with $\alpha$ or $\beta$.

The successive Kasner periods are longer as $\tau$ increases. In fact from Eqs. (25) we derive $\dot{\beta}_{0}<\dot{\beta}_{1}<0$ and $\dot{\alpha}_{1}=2\left(\dot{\alpha}_{0}+\dot{\gamma}_{0}\right)-\dot{\alpha}_{0}$. But $\dot{\alpha}_{0}+\dot{\gamma}_{0}<0$, therefore $\dot{\alpha}_{1}-\left|\dot{\alpha}_{0}\right|=2\left(\dot{\alpha}_{0}+\dot{\gamma}_{0}\right)<0$, i.e. both $\dot{\beta}_{1}$ and $\dot{\alpha}_{1}$ are absolutely smaller than $\dot{\beta}_{0}$ and $\dot{\alpha}_{0}$ respectively. Similarly from Eqs. (26) we derive $\dot{\beta}_{1}<$ $\dot{\beta}_{2}<0$ and $\dot{\gamma}_{1}<\dot{\gamma}_{2}<0$, i.e. $\dot{\beta}_{2}$ and $\dot{\gamma}_{2}$ are absolutely smaller than $\dot{\beta}_{1}$ and $\dot{\gamma}_{1}$ 


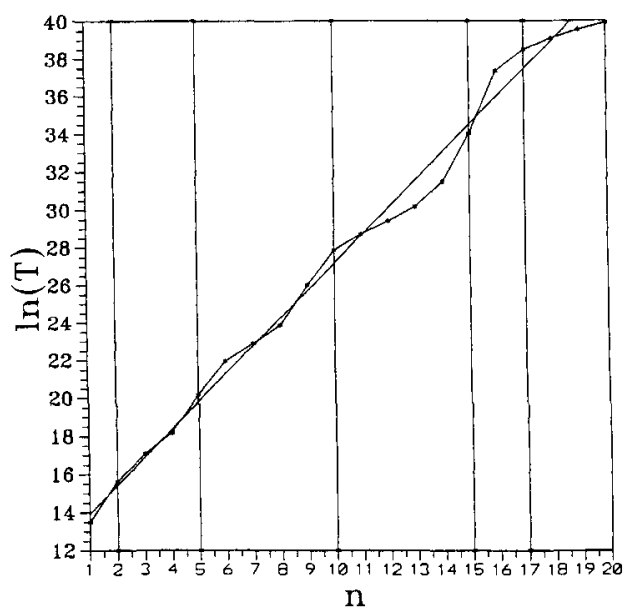

Fig. 5. The length of the Kasner periods $T$ as a function of the order $n$ (dots). The vertical lines separate the various Kasner eras. The straight line gives the best fit (Eq.27).

respectively. In every successive Kasner period similar inequalities show that $\dot{\alpha}, \dot{\beta}$, $\dot{\gamma}$ become always smaller absolutely. Thus the corresponding Kasner periods $T$ become longer. In Fig.5 we give the length of successive Kasner periods. This length increases exponentially according to the formula

$$
T=T_{n}=T_{0} e^{\kappa n}
$$

where $T_{0}=339558, \kappa=1.43121$ and $n$ is the order of the Kasner period (straight line in Fig.5). This relation implies that $T$ is proportional to $\tau$. (It is approximately $T_{n}=3 \tau_{n}$, where $\tau_{n}$ is the begining of the nth period).

In order to find the source of chaos in the Mixmaster model we solved analytically the variational equations (13) for the various Kasner periods. These give only linear deviations $\xi$ even when one variable among $\alpha, \beta, \gamma$ is close to zero. Only when two, or three variables $\alpha, \beta, \gamma$ are close to zero, we have exponential deviations of $\xi$. The main region where such exponential deviations are observed is near the maximum $(\alpha+\beta+\gamma)$, where all three quantities $\alpha, \beta, \gamma$ are close to zero. In this region the values of the local $L C N$ are different from zero (Fig. 4c), while further away they are very close to zero in general (Figs. 4a,d).

One may ask whether chaos is also produced by the variation of the number of Kasner periods during a Kasner era (Khalatnikov et al. 1985). In fact, a small change of the initial conditions produces a different sequence of Kasner periods, that leads to an exponential deviation of $\xi$ of the form

$$
\xi=\xi_{0} e^{q i}
$$

where $q$ is a constant and $i$ is the order of the Kasner era. 
But if $k_{i}$ is the number of the Kasner periods within a Kasner era, then $n=$ $k_{1}+k_{2}+\ldots+k_{i}$ and the typical length of a Kasner period during the $i$ era is

$$
T_{i}=T_{0} e^{\kappa\left(k_{1}+k_{2}+\ldots+k_{i}\right)} \approx T_{0} e^{i \kappa k}
$$

where $k$ is the average number of Kasner periods within a Kasner era.

The corresponding time $\tau$ is then

$$
\tau=k T_{0}\left(e^{\kappa k}+e^{2 \kappa k}+\ldots+e^{i \kappa k}\right) \approx k T_{0} e^{i \kappa k}
$$

and we derive

$$
i \approx \ln \tau / \kappa k \text {. }
$$

Thus $\xi$ is given by the power law in $\tau$

$$
\xi=\xi_{0} \tau^{s}
$$

where $s=q / \kappa k$, i.e. $\xi$ is not exponential in the time $\tau$. Thus this effect is not chaotic in the usual sense.

Another case, where we find exponential deviations $\xi$, is when two quantities among $\alpha, \beta, \gamma$ happen to be close to zero. Such cases are exceptional (Khalatnikov et al. 1995), and any local increase in $\xi$, that they introduce, is counteracted by the large increase of the length of the Kasner periods.

We conclude that chaos, in the sense of exponential deviation of $\xi$ in time $\tau$, is introduced mainly near the maximum of $(\alpha+\beta+\gamma)$. As a consequence, the finite time LCN is always positive. But, as $\tau$ increases considerably, the finite time LCN tends to zero and the usual LCN is zero. This behaviour is very similar to a chaotic scattering case (Contopoulos et al. 1995).

Of course a different way of measuring time may lead to a positive LCN. In fact it is well known in classical mechanics that a change of the time coordinate leads to a change of the LCN. This was established already by Poincare (1892) in the case of unstable periodic orbits. A recent discussion of the problem of different times in the Mixmaster model was made by Szydlowski (1997).

In our paper we have used only the Belinskii-Khalatnikov time $\tau$. In this time our conclusions regarding the chaotic behaviour of the Mixmaster model are clear. Further details of this problem will be given in a future paper.

\section{Chaos in General Relativity.}

A particular problem in General Relativity where chaos is predominant, is the motion of photons, or of particles of nonzero rest-mass, around two fixed black holes (Contopoulos 1990, 1991; more recent work has been done by Dettmann et al. 1994, Yurtsever 1995, and Cornish \& Gibbons 1997). 


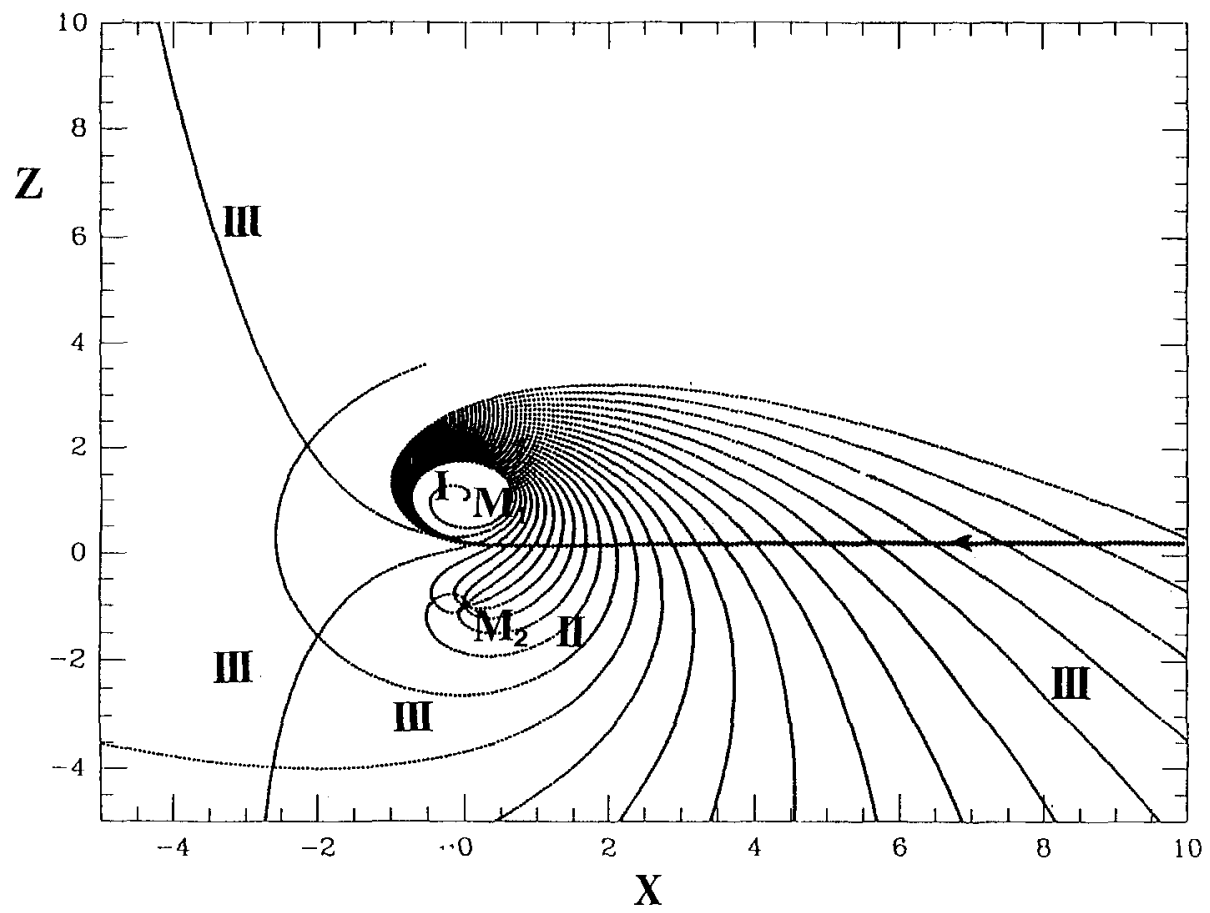

Fig. 6. A thin beam of photons from infinity is split into orbits of type I (falling to $M_{1}$ ), II (falling to $M_{2}$ ), and III (going to infinity).

This problem is remarkable because the corresponding classical problem of two fixed centers is completely integrable. The metric of the relativistic problem was given by Majumdar (1947) and Papapetrou (1947);

$$
d s^{2}=U^{-2} d t^{2}-U^{2}\left(d x^{2}+d y^{2}+d z^{2}\right),
$$

where $U$ is the potential due to two black holes of masses $M_{1}$ and $M_{2}$, written in the form

$$
U=1+\frac{M_{1}}{M_{2}}+\frac{M_{2}}{r_{2}} \text {. }
$$

We assume $M_{1}$ and $M_{2}$ to be on the $\mathrm{z}$-axis at the points \pm 1 , while the third particle is at distances $r_{1}$ and $r_{2}$ from $M_{1}$ and $M_{2}$.

A beam of photons coming from infinity (Fig.6) is separated into orbits of 3 types: (I) Orbits escaping to $M_{1}$; (II) Orbits escaping to $M_{2}$; and (III) Orbits escaping to infinity.

This is a typical scattering problem. It is chaotic because between an orbit of type I and an orbit of type II there are orbits of type III (Fig.6), between an orbit of type I and an orbit of type III there are orbits of type II and so on. This separation is 


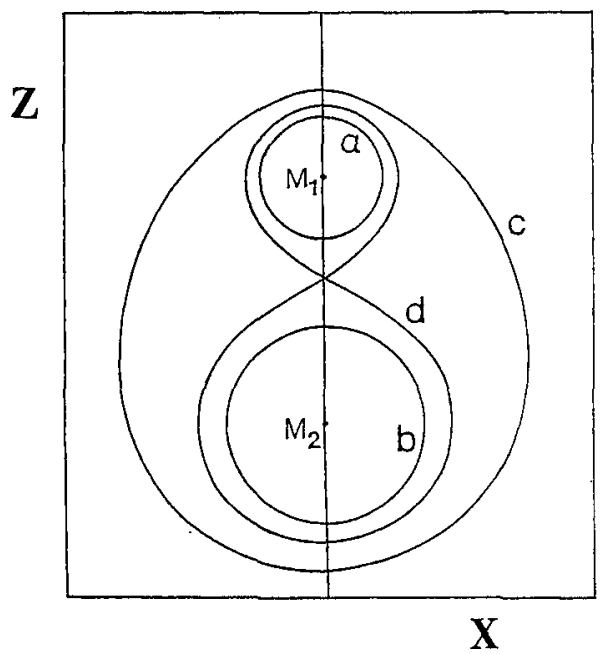

Fig. 7. Four types of unstable periodic orbits: (a) around $M_{1}$, (b) around $M_{2}$, (c) around both $M_{1}$ and $M_{2}$, and (d) figure eight orbits around both $M_{1}$ and $M_{2}$.

repeated on finer and finer scales. The orbits I,II and III form Cantor sets of finite measure (fractal sets).

Besides these orbits there are inifinite types of unstable periodic orbits that form a set of measure zero. The most important of them are the satellite orbits (a) and (b) around $M_{1}$ and $M_{2}$ respectively, and the almost elliptical orbits (c) around both $M_{1}$ and $M_{2}$ (Fig. 7).

Around each orbit of a given type (say of type II, Fig.8) there is a continuous set of similar orbits, limited by two asymptotic orbits to the same unstable periodic orbit (orbit $b$ in Fig.8). These asymptotic orbits approach the orbit $b$ from opposite directions. All continuous sets of orbits I,II and III, that are parts of the 3 fractal sets, are limited by asymptotic orbits to the orbits a, b or c respectively.

On one side of such an asymptotic orbit all nearby orbits are of the same type (e.g. orbits of type II in Fig.8), while on the other side there are infinite sets of all 3 types. Orbits close to other periodic orbits (like orbit $d$ in Fig.7, that is of figure eight type) are fractal sets of all 3 types on both their sides.

If we consider orbits starting perpendicularly to the z-axis above $M_{1}$, we find that all orbits starting inside the periodic orbit (a) fall into $M_{2}$, while all orbits outside the periodic orbit (c) go to infinity. Between the orbits (a) and (c) are the three fractal sets of orbits I,II and III.

In the case of photons there are no stable periodic orbits. However in the case of particles with non-zero rest mass there may be stable periodic orbits of various types. E.g. such orbits appear near the maximum mass $M_{1}$ of one type of orbits for fixed energy and fixed $M_{2}$. In Fig.9 we give the characteristic of the orbits of type (a) for $E=\sqrt{0.5}$ and $M_{1}=1$. This characteristic has a maximum $M_{1}=M_{1 \max }$ 


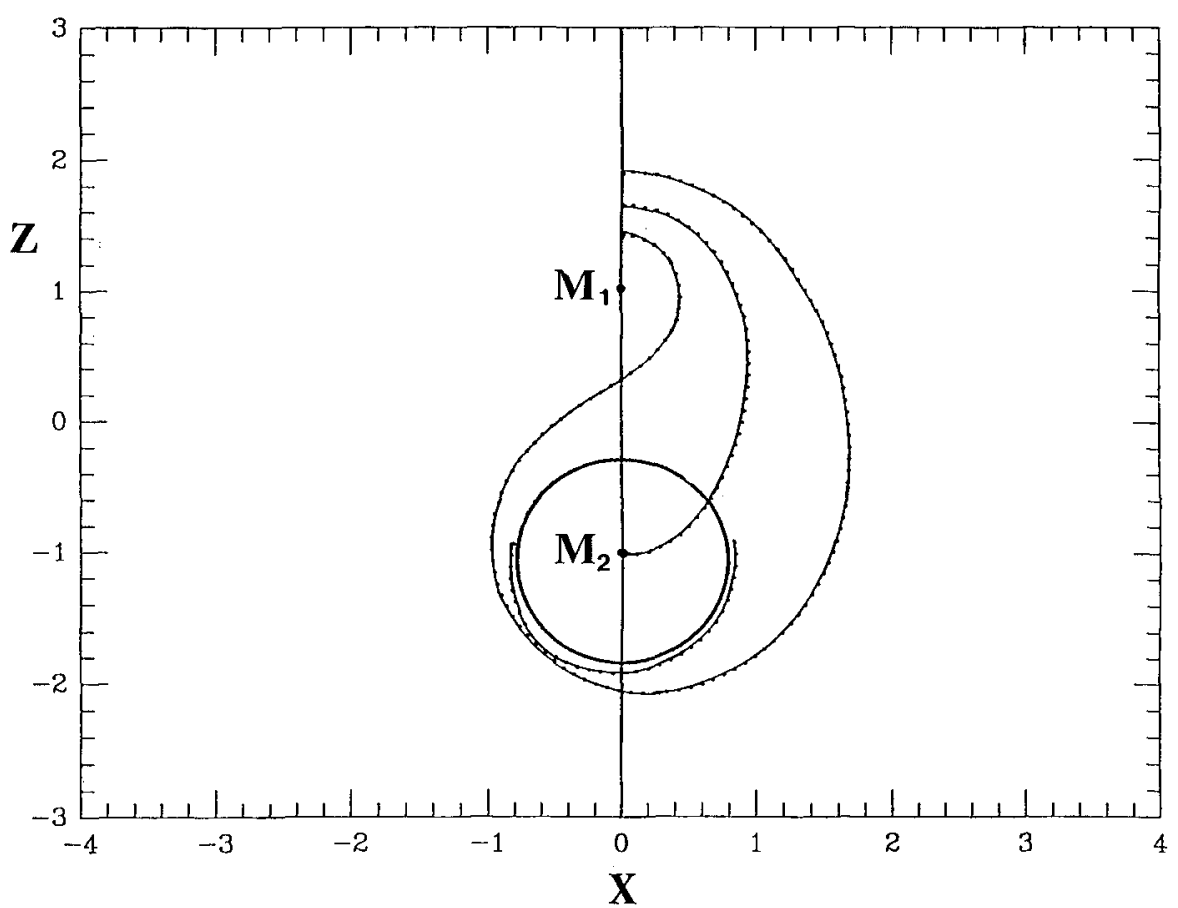

Fig. 8. A set of orbits of type II starting perpendiculary to the z-axis above $M_{1}$. The set is limited by two asymptotic curves to the unstable periodic orbit $b$.

and according to a well-known theorem of Poincaré at this maximum start two branches of orbits (a), one stable and one unstable.

In Fig. 9 we see some bifurcations of the family (a). The most important bifurcation is the one of double period (2) that appears at $M_{1}=M_{1,2}$. The double period family exists for $M_{1}$ smaller than $M_{1,2}$. It is initially stable and becomes unstable at $M_{1}=M_{1,4}$, when a period-4 bifurcation is generated. In the same way there is a period-8 bifurcation at $M_{1}=M_{1,8}$ etc.

The most important remark is that the intervals between successive bifurcations decrease almost geometrically with the universal ratio appropriate for conservative systems (Bennetin et al. 1980), namely

$$
\frac{M_{1,2}-M_{1,4}}{M_{1,4}-M_{1,8}} \approx \frac{M_{1,4}-M_{1,8}}{M_{1,8}-M_{1,16}} \approx \ldots=8.72
$$

The values of $M_{1,2^{n}}$ converge to a minimum value $M_{1, \infty}$ beyond which there is an infinity of unstable orbits of multiplicity $2^{n}(n=0,1,2, \ldots)$.

Thus in general the orbits of particles of type (a) and their bifurcations are unstable and lead to a large degree of chaos. However there are intervals of stable orbits surrounded by orbits that do not escape to $M_{1}, M_{2}$ or to infinity. The nonescaping orbits are in general ordered (quasiperiodic), but there are also some chaotic nonescaping orbits (Contopoulos 1991). 


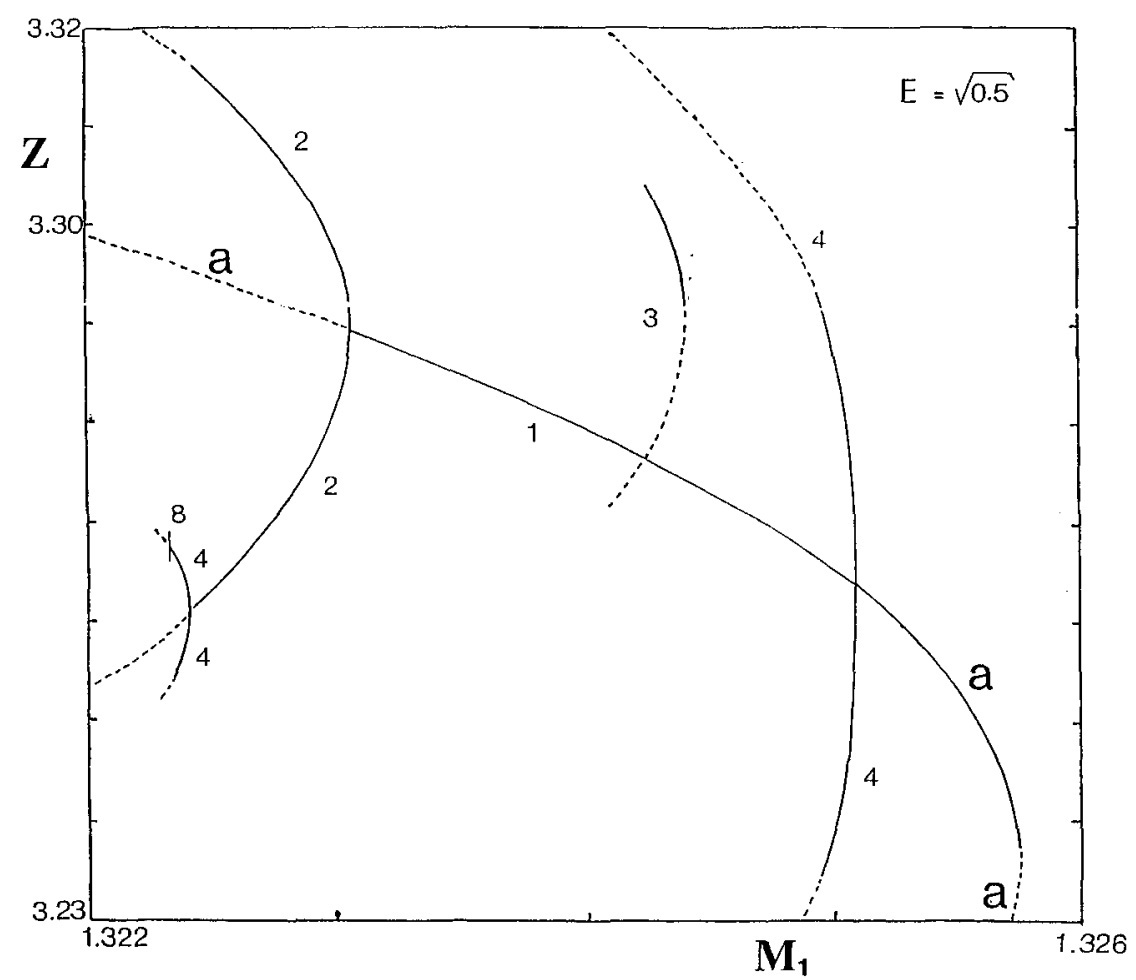

Fig. 9. Characteristics of the two families of orbits of particles of type (a) (stable and unstable) near the maximum $M_{1}$. From the stable family (a) bifurcate higher order periodic orbits. (-)stable, and (....) unstable orbits.

We conclude that chaos in the above relativistic cases has similar characteristics as chaos and chaotic scattering in classical problems. Even some numerical results are the same, as exemplified by the appearance of the same bifurcation ratio (35) in relativity as in classical dynamics.

\section{Acknowledgements}

This research was supported in part by the Academy of Athens program 200/209. C.E. was supported by the Greek Foundation of State Scholarships (IKY).

\section{References}

Ablowitz, M.J., Ramani, A. and Segur, H.:1980, J.Math.Phys.,21, 715.

Barrow, J.D., and Levin, J.: 1998, Phys. Rev. Lett., 80, 656.

Belinskii, V.A. and Khalatnikov, I.M.: 1969, Sov.Phys.JETP, $29,911$.

Benettin, G., Cercignani, C., Galgani, L. and Giorgilli, A.: 1980, Lett. Nuovo Cim., 28, 1.

Bombelli, L. and Calzetta, E.: 1992, Class. Quantum Grav., 9, 2573.

Calzetta, E. and El Hasi, C.: 1993, Class. Quantum Grav., 10, 1825.

Chicone, C., Mashhoon, B. and Retzloff, D.G.: 1997,Class. Quantum Grav., 14, 699.

Christiansen, F., Rugh, H.H. and Rugh, S.E.: 1995, J.Phys.A, 28, 657. 
Contopoulos, G.: 1990, Proc.R.Soc.Lond. A, 431, 183.

Contopoulos, G.: 1991, Proc.R.Soc.Lond. A, 435, 551.

Contopoulos, G., Grammaticos, B. and Ramani, A.: 1993, J.Phys.A,26, 5795.

Contopoulos, G., Grammaticos, B. and Ramani, A.: 1995, J.Phys.A, 28, 5313.

Cornish, N.J. and Gibbons, G.W.: 1997, Class. Quantum Grav., 14, 1865.

Cornish, N.J. and Levin, J.J.: 1997, Phys. Rev. Lett.,78, 998.

Cornish, N.J. and Levin, J.J.: 1998, Phys. Rev. D, 55, 7489.

Cornish, N.J. and Shellard, E.P.S.: 1998,Phys. Rev. Lett., 81, 3571.

Cushman, R. and Sniatycki, J.: 1995, Rep.Math.Phys., 36, 75.

Darian, B.K. and Künzle, H.P.: 1996, Class. Quantum Grav., 13, 2651.

Dettmann, C.P., Frankel, N.E. and Cornish, N.J.: 1994, Phys. Rev. D, 50, R618.

Drake, S.P., Dettmann, C.P., Frankel, N.E. and Cornish, N.J.: 1996, Phys.Rev. E,53, 1351.

Hobill, D., Bernstein, D., Simpkins, D. and Welge, M.: 1992, Class. Quantum Grav., 8, 1155.

Hobill, D., Burd, A. and Coley, A. (eds): 1994, Deterministic Chaos in General Relativity, Plenum Press, N. York.

Khalatnikov, I.M., Lifshitz, E.M., Khanin, K.M., Shchur, I.N. and Sinai, Ya.G.: 1985, J.Stat.Phys., $38,97$.

Landau, L.D., and Lifshitz, E.M.: 1971, The Classical Theory of Fields, Pergamon Press, Oxford.

Latif, A., Musette, M. and Conte, R.: 1994, Phys.Lett. A,194, 83.

Letelier, P.S. and Vieira, W.M.: 1997,Class. Quantum Grav., 14, 1249.

Majumdar, S.D.: 1947, Phys. Rev.,72, 390.

Misner, C.M.: 1969,Phys. Rev. Lett., 22, 1071.

Misner, C.M., Thorne, K. and Wheeler, J.A.: 1977, Gravitation, Freeman, San Francisco.

Papapetrou, A.: 1947, Proc. R. Irish Acad.,51, 191.

Podolsky, J. and Vesely, K. : 1998, Phys. Rev. D,58, 081501.

Poincaré, H.: 1892, Les Méthodes Nouvelles de la Mécanique Céleste, I, Gauthier Villars, Paris.

Raychaudhuri, A.: 1955, Phys.Rev., 98, 1123.

Rugh, S.E. and Jones, B.J.T.: 1990, Phys.Lett. A,147, 353.

Smith, H. and Contopoulos, G.: 1996, Astron. Astrophys., 314, 795.

Suzuki, S. and Maeda, K.: 1997,Phys. Rev. D,55, 4848.

Szydlowski, M.: 1997, Gen.Rev.Grav.,29, 185.

Taub, A.H.: 1951,Ann.Math.,53, 472.

Varvoglis, H. and Papadopoulos, D.: 1992,Astron. Astrophys.,261, 664

Voglis, N. and Contopoulos, G.: 1994, J.Phys.A,27, 4899.

Yurtsever, U.: 1995,Phys. Rev. D,52, 3176. 